\title{
Association of arsenic exposure and cognitive impairment: a population-based cross-sectional study in China
}

\section{Wang Xin}

Xiangya Hospital Central South University

Huang Xiaoyan

Xiangya Hospital Central South University

Zhou Lu

Xiangya Hospital Central South University

Chen Juan

Xiangya Hospital Central South University

\section{Zhang Xuxiang}

Xiangya Hospital Central South University

Xu Ke

Xiangya Hospital Central South University

Huang Zhijun

Xiangya Hospital Central South University

He Meian

Xiangya Hospital Central South University

\section{Shen Minxue}

Xiangya Hospital Central South University

\section{Chen Xiang}

Xiangya Hospital Central South University

\section{Tang Beisha}

Xiangya Hospital Central South University

\section{Shen Lu}

Xiangya Hospital Central South University

Yafang Zhou ( $\nabla$ zyf_1981@csu.edu.cn )

Xiangya Hospital Central South University https://orcid.org/0000-0002-1689-4462

\section{Research}

Keywords: Dementia, Cognitive impairment, Arsenic, Arsenicosis, Cross-sectional study, Risk factor 
Posted Date: March 30th, 2020

DOl: https://doi.org/10.21203/rs.3.rs-19627/v1

License: (c) (i) This work is licensed under a Creative Commons Attribution 4.0 International License. Read Full License 


\section{Abstract}

Background: Chronic arsenic exposure is a major public health issue worldwide. Previous studies explored the effects of arsenic exposure on cognitive impairment, but majority of previous studies focused on the effects on school children rather than adults. Moreover, few studies investigated the role of arsenic exposure in cognitive impairment process in a large scale in China.

Methods: Aimed to evaluate the association between long-term exposure to arsenic and cognitive impairment in China, we designed a cross-sectional analytical study in which 1,556 adults from three locations around the Realgar Plant (Heshan Village, Wangyangqiao Community and Baiyangshan Village) were enrolled. Participants' general cognitive function were evaluated by using a Chinese version of Mini-mental state Examination (MMSE). Internal arsenic exposure status of participants (hair arsenic concentrations) was measured by inductively coupled plasma-mass spectroscopy (ICP-MS), and external arsenic exposure status of participants was approximately evaluated by measuring the distance between the participants' location to Realgar Plant.

Results: In our study, we found that the prevalence of cognitive impairment in arsenic-endemic areas was $20.6 \%$, which was significantly higher than the prevalence of cognitive impairment in China (14.5\%). Hair arsenic concentrations and prevalence of arsenicosis in cognitive impaired group $(\mathrm{Cl})$ was significantly higher than those in cognitive normal group $(C N)(P<0.05)$.

Conclusions: Our analyses revealed that the distance away from Realgar Plant was positively correlated with MMSE scores, while inversely associated with the prevalence of cognitive impairment. In addition, we found that MMSE scores were decreased with the increasing hair arsenic concentrations in a dosedependent manner. In two-level Logistic regression analysis, arsenicosis was still a risk factor for cognitive impairment (odds ratio $(O R)=1.77, P<0.05$ ) even after adjusting for potential confounding variables. Our results showed that chronic arsenic exposure decreased cognitive function in adults in a dose-dependent manner, and arsenicosis was a risk factor for cognitive impairment.

\section{Background}

As global aging population increase dramatically [1], the aging and age-related diseases such as dementia have become the focus of attention. In China, it was estimated that 9.5 million people over age 65 years were suffering from dementia [2]. Dementia imposed a heavy financial burden on patients and their families. The per capita annual cost was US\$19,144.36 and the national annual costs was US\$167.74 billion in China in 2015 [3].

Arsenic (As), a toxic metalloid element widely distributed in nature, has been found to be the risk factor for a variety of diseases including hypertension, atherosclerosis, type-two diabetes, myocardial infarction, keratosis, anemia, skin cancer, bladder cancer, lung cancer, and cognitive impairment [4]. Long-term exposure to air, soil, water and food with high concentration of arsenic leads to a chronic systemic illness called arsenicosis. Arsenicosis is a global public health issue. Tens of millions of people worldwide are at 
risk of exposure to arsenic, including the United States, Chile, Bangladesh, Thailand, China, India, Mexico and Hungary [5]. Several epidemiological researches showed the association between chronic arsenic exposure and cognitive impairment [6-9]. However, majority of previous researches of arsenic exposure and cognitive function focused on the effects of arsenic on school children's cognition rather than adults and elders, which led to restriction in the applicability of the findings. Moreover, previous studies used groundwater arsenic concentrations as an indicator of arsenic exposure levels, and lack of appropriate markers for reflecting actual individual arsenic exposure levels precisely.

Realgar Plant, located in Shimen County, Hunan Province of central south China, was known as the largest Realgar Plant in Asia with a mining history of more than 1500 years [10]. It was intensively mined and smelted since the 1950s, until it was shut down by the government due to the serious environmental pollution in 2011 [10, 11]. In 1994, the peak level of arsenic in local drinking water was 14,531 $\mu \mathrm{g} / \mathrm{L}$, and it substantially dropped to $765 \mu \mathrm{g} / \mathrm{L}$ in 2014, which still exceeded the World Health Organization (WHO) standard $(0.01 \mathrm{mg} / \mathrm{L})[11,12]$. Despite the improvement of local environment under the government's guidance and supervision, the soil and groundwater seriously contaminated by arsenic continually endanger local residents. To investigate the correlation between arsenic exposure and cognition function in a large sample of adults, we conducted a cross-sectional study in the residents around Realgar Plant who had long-term exposure to arsenic.

\section{Methods}

\section{Selection of study areas and participants}

A population-based cross-sectional survey was performed in three locations around Realgar Plant (Heshan Village, Wangyangqiao Community and Baiyangshan Village) between January to March 2019 in Shimen County, Hunan Province of China. Heshan Village, Wangyangqiao Community and Baiyangshan Village is $2 \mathrm{~km}, 5 \mathrm{~km}, 10 \mathrm{~km}$ away from Realgar Plant, respectively (Fig.1). The livelihoods of the residents living in the three locations were approximately similar. Because the lack of data of external arsenic exposure level (arsenic concentrations of atmosphere, soil, water, etc.), the distance from the location to Realgar Plant can be used as representation for the previous level of arsenic exposure approximately. Participants were recruited from a medical examination program approved by the local government at Realgar Plant Hospital. Written informed consent forms were obtained prior to the present study from all participants. The individuals ( $\geq 18$-year-old) who had lived in these three locations and owning the local household registration were recruited for this study. Individuals with hearing or vision difficulties, neurological conditions, severe mental disorders or end-stage physical diseases (e.g., Parkinson's disease, epilepsy, multiple sclerosis, stroke, schizophrenia, depressive disorder, heart failure, liver failure, respiratory failure, uremia, etc.) were excluded. The demographic characteristics of participants collected by questionnaire included: age, sex, height, weight, location, body mass index (BMI), education, personal medical history, and diastolic blood pressure (DBP), and systolic blood pressure (SBP). 


\section{Collection of blood sample and measurement of biochemical index}

Fasting blood samples were collected between 8 to 9 a.m. Samples were stored in a cold-chain box and sent to Shimen People's Hospital to test fasting blood glucose and blood lipids. Indices of blood lipids examination included: total cholesterol (TC), high density lipoprotein cholesterol (HDL-C), low density lipoprotein cholesterol (LDL-C), and triglyceride (TG). Among the 1556 participants, 294 participants were unavailable or declined to provide blood samples. The total number of blood samples used for the biochemical index determination was thus 1262 .

\section{Cognitive function and sleep assessments}

The general cognitive function was evaluated by using a Chinese version of MMSE which ranged from 0 to 30. The higher MMSE scores suggest the better cognitive function. As MMSE scores are susceptible to educational level, the cut-off score indicating cognitive impairment for different education levels are: $\leq 17$ points for illiterate participants, $\leq 20$ points for participants accepted primary education and $\leq 24$ points for participants accepted secondary or higher education [13].

Sleep quality was assessed by Pittsburgh sleep quality index (PSQI). PSQI is a well-validated tool aimed to evaluate subjective sleep quality. It is a questionnaire with 19 self-related items, composed of 7 subsections: subjective sleep quality, sleep duration, sleep latency, sleep efficiency, sleep disturbances, usage of sleeping pills, and daytime dysfunction. Each subsection score ranges from 0 to 3 , and the total score of PSQI is 21. A lower PSQI score indicated better sleep quality [14].

All assessments were conducted by trained researchers in the field of neurology according to the protocol. All of the researches were blind to the study areas and cognitive status of participants.

\section{Arsenic exposure status assessment and diagnosis of endemic arseniasis}

Previous study suggested that arsenic concentrations in hair samples can be used as an effective marker of arsenic intake [15]. Therefore, in this study, we collected hair samples (approximately $1 \mathrm{~cm}$ in length) behind the ear and sealed them separately in envelopes. Samples were sent to the local Occupation Disease Prevention and Treatment Center for analyzing. The accurate arsenic concentrations in hair samples were measured by inductively coupled plasma-mass spectroscopy (ICP-MS) [15].

Endemic arsenicosis was diagnosed according on the diagnosis standards of medical and health professions formulated by National Health Commission of the People's Republic of China (PRC) in 2015. Residents of endemic arsenicosis areas can be diagnosed as arsenicosis if they meet one of the following clinical characteristics: (i) existing unexplained papular, nodular or verrucous hyperkeratosis on the skin of palms and soles, or (ii) existing diffuse or scattered pigmentation spots and/or round depigmentation spot of varying sizes with blur edges on the skin of trunk or non-exposed parts. Meanwhile, arsenic concentrations in hair or urine samples were significantly higher than the normal reference values in non-endemic areas can be used as a reference indicator for the diagnosis of arsenicosis [16]. The arseniasis participants were unanimously diagnosed by two dermatologists in 
Xiangya Hospital and Centers for Disease Control (CDC) according to the protocol. All of the researchers were blind to the study areas and cognitive status of participants.

\section{Statistical Analysis}

Data from all questionnaires were processed by SPSS. Characteristics of participants were described using mean \pm standard error for normal distributed continuous variables or median ( $25 \%$ quartile, $75 \%$ quartile) for skewed distributed continuous variables, and frequency (\%) for categorical variables. When the sample distribution conformed to normality, differences between two continuous variables groups were assessed by using Student's t test. For the two categorical variables groups comparisons, Chisquare test was used to analyze the differences. Differences among means of multiple groups were assessed using one-way analysis of variance (ANOVA). For skewed distributed variables, a nonparametric test was used. Two-level Logistic regression analysis was used to determine whether expose to arsenic was a risk factor for cognitive impairment, results were presented as OR and adjusted OR with 95\% confidence intervals $(\mathrm{Cl})$. We performed linear regression analysis to examine the association of the hair arsenic concentrations and MMSE scores. All analyses were performed using SPSS version 25.0 (SPSS Inc., Chicago, IL, USA). Comparisons between groups were considered to be statistically significant when $\mathrm{p}<0.05$.

\section{Results}

A total of 1556 participants, including 802 males and 754 females, were included in our study. $\mathrm{Cl}$ prevalence of females was significantly higher than that of males ( $24.8 \%$ vs $16.7 \%, P<0.05)$. The average age of participants was $57.0 \pm 11.5$ years. The percentages of participants from Heshan Village, Wangyangqiao Community and Baiyangshan Village were $48.2 \%, 36.1 \%$ and $15.7 \%$, respectively. There were 321 (20.6\%) participants in $\mathrm{Cl}$ and 1235 (79.4\%) in $\mathrm{CN}$ included in our study. In $\mathrm{Cl}$, the proportions of participants without education and with primary education, secondary or higher education were $47.6 \%$, $18.9 \%$ and $15.5 \%$, respectively. In $\mathrm{CN}$, the proportions were $52.4 \%, 81.1 \%$ and $84.5 \%$, respectively. There was a statistically significant difference between $\mathrm{Cl}$ and $\mathrm{CN}$ groups in the education levels $(P<0.05)$. In other words, the illiteracy participants were most likely prone to cognitive impairment, and participants accepted secondary or higher education had the lowest prevalence of cognitive impairment. MMSE score of $\mathrm{Cl}$ was significantly lower than that of $\mathrm{CN}(16.6 \pm 5.47$ vs. $26.3 \pm 2.81, \mathrm{P}<0.05)$, and $\mathrm{PSQI}$ score in $\mathrm{Cl}$ was $6.48 \pm 3.89$ and in $\mathrm{CN}$ was $5.71 \pm 3.99$, significantly higher than that of $\mathrm{CN}(P<0.05)$. Hair arsenic concentrations (HAs) in $\mathrm{Cl}$ was significantly higher than $\mathrm{CN}(0.27(0.14,0.56) \mathrm{mg} / \mathrm{kg}$ vs. $0.20(0.10,0.41)$ $\mathrm{mg} / \mathrm{kg}, \mathrm{P}<0.05)$. The prevalence of arsenicosis in all participants was $49.2 \%$. The prevalence of arsenicosis in $\mathrm{Cl}$ was higher than $\mathrm{CN}(64.5 \%$ vs. $45.3 \%, \mathrm{P}<0.05)$. The demographic characteristics of the subjects was shown in Table 1.

Table 1 Demographic characteristics for participants 


\begin{tabular}{|c|c|c|c|c|}
\hline & Overall $\mathrm{N}=1556$ & CI & $\mathrm{CN}$ & $P$ value \\
\hline & & $\mathrm{N}=321$ & $\mathrm{~N}=1235$ & \\
\hline Sex, Female, N (\%) & 754 (48.5) & $187(24.8)$ & $567(75.2)$ & $<0.001$ \\
\hline Age, year & $57.0 \pm 11.5$ & $63.4 \pm 10.6$ & $55.3 \pm 11.1$ & $<0.001$ \\
\hline Residence Locations, N (\%) & & & & 0.012 \\
\hline Heshan Village & 750 (48.2) & 177 (23.6) & $573(76.4)$ & \\
\hline Wangyangqiao Community & $561(36.1)$ & $107(19.1)$ & $454(80.9)$ & \\
\hline Baiyangshan Village & 245 (15.7) & $37(15.1)$ & $208(84.9)$ & \\
\hline BMI & $23.9 \pm 3.41$ & $23.7 \pm 3.20$ & $24.0 \pm 3.45$ & 0.164 \\
\hline Education level, N (\%) & & & & $<0.001$ \\
\hline Illiteracy & $168(10.8 \square$ & $80(47.6)$ & $88(52.4 \square$ & \\
\hline Primary & 756 (48.6) & $143(18.9)$ & $613(81.1)$ & \\
\hline Secondary or higher & $632(40.6)$ & 98 (15.5) & $534(84.5)$ & \\
\hline \multicolumn{5}{|l|}{ Medical history, N (\%) } \\
\hline Hypertension & $423(27.2)$ & $110(34.3)$ & $313(25.3)$ & 0.001 \\
\hline Myocardial infarction & $266(17.1)$ & $83(25.9)$ & $183(14.8)$ & $<0.001$ \\
\hline Osteoporosis & $76(4.9)$ & $18(5.6)$ & $58(4.7)$ & 0.500 \\
\hline SBP (mmHg) & $146 \pm 19.9$ & $151 \pm 19.6$ & $144 \pm 19.7$ & $<0.001$ \\
\hline DBP (mmHg) & $88.7 \pm 12.5$ & $89.1 \pm 11.4$ & $88.6 \pm 12.7$ & 0.246 \\
\hline Fasting glucose (mmol/L) & $6.35 \pm 1.90$ & $6.55 \pm 1.73$ & $6.29 \pm 1.95$ & $<0.001$ \\
\hline \multicolumn{5}{|l|}{ Blood lipid } \\
\hline $\mathrm{TC}(\mathrm{mmol} / \mathrm{L})$ & $5.18 \pm 1.02$ & $5.20 \pm 1.03$ & $5.17 \pm 1.02$ & 0.646 \\
\hline HDL-C (mmol/L) & $1.41 \pm 0.33$ & $1.42 \pm 0.32$ & $1.41 \pm 0.34$ & 0.652 \\
\hline LDL-C (mmol/L) & $3.18 \pm 0.86$ & $3.22 \pm 0.91$ & $3.17 \pm 0.84$ & 0.472 \\
\hline TG $(\mathrm{mmol} / \mathrm{L})$ & $1.44 \pm 1.24$ & $1.50 \pm 1.38$ & $1.42 \pm 1.19$ & 0.485 \\
\hline MMSE & $24.3 \pm 5.30$ & $16.6 \pm 5.47$ & $26.3 \pm 2.81$ & $<0.001$ \\
\hline Instant memory & $2.64 \pm 0.78$ & $1.86 \pm 1.17$ & $2.85 \pm 0.46$ & $<0.001$ \\
\hline Delayed memory & $2.00 \pm 1.51$ & $0.77 \pm 1.04$ & $2.32 \pm 0.94$ & $<0.001$ \\
\hline Orientation & $9.05 \pm 1.43$ & $7.45 \pm 2.21$ & $9.47 \pm 0.68$ & $<0.001$ \\
\hline
\end{tabular}




\begin{tabular}{|c|c|c|c|c|}
\hline Visuospatial ability & $0.57 \pm 0.49$ & $0.26 \pm 0.44$ & $0.66 \pm 0.48$ & $<0.001$ \\
\hline Executive ability & $2.52 \pm 0.74$ & $1.92 \pm 1.02$ & $2.67 \pm 0.56$ & $<0.001$ \\
\hline Language ability & $7.31 \pm 1.85$ & $5.12 \pm 2.18$ & $7.88 \pm 1.23$ & $<0.001$ \\
\hline Attention and arithmetic & $3.31 \pm 1.68$ & $1.36 \pm 1.39$ & $3.81 \pm 1.34$ & $<0.001$ \\
\hline PSQI & $5.89 \pm 3.98$ & $6.48 \pm 3.89$ & $5.71 \pm 3.99$ & 0.002 \\
\hline subjective sleep quality & $1.09 \pm 0.78$ & $1.24 \pm 0.72$ & $1.05 \pm 0.79$ & $<0.001$ \\
\hline sleep latency & $2.04 \pm 2.10$ & $2.34 \pm 2.20$ & $1.96 \pm 2.07$ & 0.004 \\
\hline sleep duration & $0.35 \pm 0.78$ & $0.32 \pm 0.72$ & $0.36 \pm 0.79$ & 0.582 \\
\hline sleep efficiency & $0.71 \pm 1.01$ & $0.80 \pm 1.02$ & $0.68 \pm 1.01$ & 0.060 \\
\hline sleep disturbances & $0.77 \pm 0.42$ & $0.79 \pm 0.41$ & $0.77 \pm 0.42$ & 0.386 \\
\hline use of sleeping medicine & $0.02 \pm 0.21$ & $0.02 \pm 0.19$ & $0.02 \pm 0.22$ & 0.757 \\
\hline daytime dysfunction & $0.90 \pm 0.83$ & $0.97 \pm 0.77$ & $0.89 \pm 0.85$ & 0.022 \\
\hline $\operatorname{HAs}\left(\mathrm{M},\left(\mathrm{P}_{25}, \mathrm{P}_{75}\right)\right)$ & $0.21(0.10,0.43)$ & $0.27(0.14,0.56)$ & $0.20(0.10,0.41)$ & $<0.001$ \\
\hline Arseniasis, N (\%) & $766(49.23)$ & $207(64.5)$ & $559(45.3)$ & $<0.001$ \\
\hline
\end{tabular}

Arsenic exposure levels and cognitive status of the participants in each village were separately shown in Table 2. We found that prevalence of arseniasis and cognitive impairment were the highest in participants from Heshan Village $(58.5 \%, 23.6 \%)$, followed by Wangyangqiao Community $(46.3 \%, 19.1 \%)$ and Baiyangshan Village $(27.4 \%, 15.1 \%)$ (Table 2$)$, which were inversely correlated with the distance between the place of residence and the pollution source. There were statistical differences among these three locations in the prevalence of arseniasis and cognitive impairment $(P<0.05)$. Additionally, MMSE scores were $24.7 \pm 4.50,24.3 \pm 5.36$ and $24.2 \pm 5.5$, in participants from Baiyangshan Village, Wangyangqiao Community and Baiyangshan Village, respectively. However, we found that hair arsenic concentrations of participants from Wangyangqiao Community was higher than that of Heshan Village and Baiyangshan Village, which could be explained by introducing survivor effect. Among the three locations, Heshan Village was the most polluted and many residents died of arseniasis in the past few decades [11]. The existing people may be more resistant to absorb arsenic, which was evidenced by the results of our study. In our study, the very low value of hair arsenic concentrations $(<0.01 \mathrm{mg} / \mathrm{kg})$ was most in Heshan Village (14.9\%), while in Wangyangqiao Community and Baiyangshan Village were $0.03 \%$ and $1.61 \%$, respectively. On the basis of above, we found that the prevalence of cognitive impairment was the highest in participants from Heshan Village where participants were highest levels of arsenic exposure. Because the lack of data of external arsenic exposure level, we speculated that there was a negative correlated 
between the distance away from the Realgar Plant and arsenic external exposure level, which was proved to be correct by these above results.

Table 2 Comparison of cognitive impairment in three locations

\begin{tabular}{|c|c|c|c|c|c|}
\hline \multirow{3}{*}{$\begin{array}{l}\text { Index } \\
\text { Distance }\end{array}$} & & & locations & & \multirow{3}{*}{$\mathrm{P}$} \\
\hline & Overall & Heshan & Wangyangqiao & Baiyangshan & \\
\hline & & $2 \mathrm{~km}$ & $5 \mathrm{~km}$ & $10 \mathrm{~km}$ & \\
\hline $\operatorname{HAs}\left(\mathrm{M},\left(\mathrm{P}_{25}, \mathrm{P}_{75}\right)\right)$ & $0.21(0.10,0.43)$ & $0.20(0.06,0.47)$ & $0.25(0.14,0.45)$ & $0.20(0.12,0.32)$ & $<0.001$ \\
\hline Arseniasis, N (\%) & $766(49.2)$ & $439(58.5)$ & $260(46.3)$ & $67(27.4)$ & $<0.001$ \\
\hline MMSE & $24.3 \pm 5.30$ & $24.2 \pm 5.49$ & $24.3 \pm 5.36$ & $24.7 \pm 4.50$ & 0.381 \\
\hline Cognitive impairment, N (\%) & $321(20.6)$ & $177(23.6)$ & 107(19.1) & $37(15.1)$ & 0.009 \\
\hline
\end{tabular}

We illustrated the dose-response relationship between arsenic exposure and MMSE scores. We performed linear regression analysis to location-stratified analysis to assess the association between hair arsenic concentrations and MMSE scores. Results shown that MMSE scores decreased with increasing hair arsenic concentrations (Fig.2).

Chi-square test was used to calculate the number of participants with cognitive impairment per quartile of hair arsenic concentrations (Table 3). Hair arsenic concentrations and cognitive impairment were included in the binary regression model, and the correlation between the level of arsenic exposure and cognitive impairment was tested. Correlation between the first quartile of hair arsenic concentrations (Q1) and prevalence of cognitive impairment was used as the reference group, and the others quartile of hair arsenic concentrations (Q2-Q4) were estimated, which expressed as OR values. We adjusted age, sex, education and location. The results revealed a positive correlation between hair arsenic concentration and prevalence of cognitive impairment. There was still a dose-response relationship after adjusting for confounding factors.

Table 3 The prevalence of cognitive impairment in arsenic inter-quartile range

\begin{tabular}{cccccc}
\hline HAs & CI, N (\%) & OR (95\% CI) & P & aOR (95\% CI) * & P \\
\hline Q1 & $56(14.4)$ & 1 & & 1 & \\
Q2 & $76(19.5)$ & $1.444(0.989,2.107)$ & 0.057 & $1.454(0.965,2.189)$ & 0.073 \\
Q3 & $87(22.7)$ & $1.713(1.183,2.480)$ & 0.004 & $1.468(0.980,2.199)$ & 0.063 \\
Q4 & $102(26.2)$ & $2.113(1.471,3.036)$ & 0.000 & $1.582(1.034,2.421)$ & 0.035 \\
\hline
\end{tabular}

*Adjusted for age, sex, education, location

Two-level Logistic regression analysis showed that arseniasis was a risk factor for cognitive impairment $(\mathrm{OR}=2.20, \mathrm{P}<0.05)$. After adjusting for age, sex, education, and location, the association attenuated but remained statistically significant $(O R=1.77, P<0.05)$. The results were shown in Table 4. 
Table 4 Two-level Logistic regression analysis

\begin{tabular}{cccccc}
\hline Model & $\mathrm{b}$ & $\mathrm{S}_{\mathrm{b}}$ & Wald $\chi 2$ & $\mathrm{P}$ & OR $(95 \% \mathrm{CI})$ \\
& & & & & \\
& & & & & \\
\hline Crude & 0.78 & 0.130 & 36.671 & 0.000 & $2.196(1.702,2.832)$ \\
Adjusted $^{*}$ & 0.570 & 0.156 & 13.420 & 0.000 & $1.768(1.303,2.398)$ \\
\hline
\end{tabular}

*Adjusted for age, sex, education, location

\section{Discussion}

The major finding of this cross-sectional study was that arsenic exposure was associated with cognitive impairment in adults and arseniasis was an independent risk factor for cognitive impairment. In addition, participants with higher hair arsenic concentrations tend to have lower MMSE scores and higher risks of cognitive impairment. Our analyses showed that the prevalence of cognitive impairment in our study was $20.6 \%$, which was significantly higher than the $14.5 \%$ prevalence of cognitive impairment in individuals in China [17]. Therefore, we infer that the arsenic pollution in Shimen country was still serious and had a great impact on the health of local residents. Even after many years of environmental improvement, most of the surrounding villagers still had cognitive impairment.

Environmental exposure to arsenic had been posited to be associated with cognitive impairment in previous studies. Tsai et al examined 49 junior school students living in arsenic exposure area and found that memory and executive functioning were significantly affected by long-term arsenic exposure [6]. In a sample of 80 school children between the ages of 6 to 9 in Mexican, Calderon et al found that arsenic concentration was inversely correlated with verbal comprehension and long-term memory [7]. O'Bryant et al analyzed 434 rural-dwelling adults and elders in a community-based study, found that low-level arsenic exposure can lead to poorer global cognition, including executive function and memory [8]. A crosssectional study conducted in Bangladesh revealed that arsenic exposure exerted a detrimental impact on intellectual functions in adolescence [9]. In animal studies, Finley and colleagues found that adolescence mice exhibited poorer memory and learning skill after perinatal arsenic exposure [18].

Our analysis shown that cognitive impairment can be considered to be affected by age, sex, education, fasting blood glucose, and SBP. Females was more likely to experience cognitive impairment than males which was consistent with previous studies [19]. Cognitive impairment had tendency to older age, lower education level, which proved that both were the main risk factors for cognitive impairment. $\mathrm{Cl}$ had higher fasting blood glucose than $\mathrm{CN}$, for hyperglycemia damaged on cognitive function [20-24]. $\mathrm{Cl}$ had higher SBP than CN in this study. A Meta-analysis showed a correlation between SBP and AD, meanwhile no correlation for DBP [25], which was consistent with our research. Our study found that $\mathrm{Cl}$ had a higher PSQI score than CN, which represented a worse quality of sleep. There are many studies that linked 
cognitive impairment to sleep, and recent article published in Science showed that both A $A$-amyloid and Tau proteins increased in the brain of healthy adults who did not sleep for one night [26].

Many literatures described the mechanisms of arsenicosis causing cognitive impairment in detail. First, arsenic inhibited the excitatory synaptic transmission in hippocampal slices of rats [27]. Second, arsenic may evoke inflammatory responses and oxidative stress via up-regulation of a range of inflammatory signaling molecules, such as tumor necrosis factor-a (TNF-a), interleukins (ILs) - IL-6, IL-8, IL-12 and nuclear factor-KB (NF-KB) [28], and impair the ubiquitin-proteasomal protein degradation system, all of which played a key roles in Alzheimer's disease (AD) [29]. Third, arsenic exposure increased tau hyperphosphorylation levels [30], interfered the expression and processing of amyloid precursor protein (APP) in neuronal cells [31], and induced the neuronal apoptosis and necrosis [28, 32, 33]. All of these supported that arsenic exposure could increase the risk of cognitive impairment.

Our research was consistent with and extend previous work to adults living in arsenic exposed areas, and the first large-scale population study explored the correlation between arsenic exposure and cognitive function in arsenic-exposed area in China. Cognitive impairment may be an important symptom of neglect in people exposed to arsenic. Our current research differed from previous studies in the impact of arsenic exposure on cognitive function in adults and elders living in three arsenic exposure communities rather than school children. Our study demonstrated that arseniasis was associated with poorer cognitive function. We showed that arseniasis was an independent risk factor of cognitive impairment. There were still some limitations in this present study. First, this study was a cross-sectional study that lacked longitudinal data, which means that we cannot infer the temporal relationship of arsenic exposure with cognitive function. Second, diagnosis of cognitive impairment was mainly based on MMSE. Although MMSE is a well-validated screening tool for cognitive impairment, it can be influenced by external factors such as age, sex, education, and cultural background. Therefore, we used a Chinese version of MMSE, and the researchers were well trained. Third, this study lacked data about external arsenic exposure levels (atmospheric, soil, water). However, we used the distance between the participants' location and Realgar Plant as a marker of external arsenic exposure status approximately.

\section{Conclusions}

In summary, our study was a large population-based study showed that arsenic exposure was associated with cognitive impairment, and hair arsenic concentrations were inversely associated with MMSE scores in a dose-dependent manner. Cognitive impairment may be an important symptom of neglect in people exposed to arsenic. As prevention was critical for dementia, our finding that arseniasis is an independent risk factor for cognitive impairment, can help prevent and delay the onset of dementia.

\section{Abbreviations}

MMSE: Mini-mental state Examination; ICP-MS: inductively coupled plasma-mass spectroscopy; Cl: cognitive impaired group; CN: cognitive normal group; OR: odds ratio; WHO: World Health Organization; 
BMI: body mass index; DBP: diastolic blood pressure; SBP: systolic blood pressure; TC: total cholesterol; HDL-C: high density lipoprotein cholesterol; LDL-C: low density lipoprotein cholesterol; TG: triglyceride; PSQI: Pittsburgh sleep quality index; PRC: the People's Republic of China; CDC: Centers for Disease Control; ANOVA: one-way analysis of variance; TNF-a冈tumor necrosis factor-a; ILs: interleukins; NF-KB: nuclear factor-KB; AD: Alzheimer's disease; APP: amyloid precursor protein

\section{Declarations}

Ethics approval and consent to participate: All procedures were approved by the Ethics Committee of Xiangya Hospital, Center South of University, and performed in accordance with Helsinki Declaration.

Consent for publication: All participants had given consent for their data to be used for research purposes.

Availability of data and materials. The datasets used and/or analyzed during the current study are available from the corresponding author on reasonable request.

Competing interests: All authors declare that they have no competing interests.

Funding. The work was supported by the Ministry of Science and Technology of China (2016YFC0900802, 2015FY111100), National Natural Science Foundation of China (No.81671075, No.81701134), the National Key R\&D Program of China (No.2017YFC0840100 and No.2017YFC0840104), and the Xiangya Hospital Youth Scientific Research Fund (No.2016Q01 to 382).

Authors' contributions: W.X, H.X.Y collected and analyzed the data, wrote the manuscript. W.X, H.X.Y, Z.L, C.J, Z.X.X, X.K, H.Z.J collected participants data from different locations. H.M.A performed the data analysis. S.M.X, C.X, T.B.S. and S.L discussed the results and commented on the manuscript. Z.Y.F conceived the project, designed research, supervised the data collection and analysis, and revised the manuscript.

Acknowledgements: We were very grateful to all of the participants who were enrolled in the study.

\section{References}

1. Nations, U. World Population Prospects. 2019. https://population.un.org/wpp. Accessed 19 Jun 2019.

2. Wu YT, Ali GC, Guerchet M, Prina AM, Chan KY, Prince M, et al. Prevalence of dementia in mainland China, Hong Kong and Taiwan: an updated systematic review and meta-analysis. Int J Epidemiol, 2018; 47(3): 709-19.

3. Jia J, Wei C, Chen S, Li F, Tang Y, Qin W, et al. The cost of Alzheimer's disease in China and reestimation of costs worldwide. Alzheimers Dement 2018, 14(4):483-91. 
4. Kapaj S, Peterson H, Liber K, Bhattacharya P. Human health effects from chronic arsenic poisoning-a review. J Environ Sci Health A Tox Hazard Subst Environ Eng 2006, 41(10):2399-428.

5. Smith, $\mathrm{AH}$; Steinmaus, $\mathrm{CM}$. Health effects of arsenic and chromium in drinking water: recent human findings. Annu Rev Public Health 2009, 30:107-22.

6. Tsai, SY, Chou, HY, The, HW, Chen, CM, Chen, CJ. The effects of chronic arsenic exposure from drinking water on the neurobehavioral development in adolescence. Neurotoxicology 2003, 24(45):747-53.

7. Calderon J, Navarro ME, Jimenez-Capdeville ME, Santos-Diaz MA, Golden A, Rodriguez-Leyva I, et al. Exposure to arsenic and lead and neuropsychological development in Mexican children. Environ Res 2001, 85(2):69-76.

8. O'Bryant SE, Edwards M, Menon CV, Gong G, Barber R. Long-term low-level arsenic exposure is associated with poorer neuropsychological functioning: a Project FRONTIER study. Int J Environ Res Public Health 2011, 8(3):861-74.

9. Wasserman GA, Liu X, Parvez F, Chen Y, Factor-Litvak P, Lolacono NJ, et al. A cross-sectional study of water arsenic exposure and intellectual function in adolescence in Araihazar, Bangladesh. Environ Int 2018, 118:304-13.

10. Tang J, Liao Y, Yang Z, Chai L, Yang W. Characterization of arsenic serious-contaminated soils from Shimen realgar mine area, the Asian largest realgar deposit in China. Journal of Soils and Sediments 2016, 16(5):1519-28.

11. Wang Z, He H, Yan Y, Wu C. Arsenic exposure of residents in areas near Shimen arsenic mine. Journal of hygiene research 1999, 28(1):12-4.

12. Hu YH, Zhou L, Li X, Xu FD, Wang L, Mo DZ, et al. Arsenic Contamination in Shimen Realgar Mine 区:As Spatial Distribution, Chemical Fractionations and Leaching. Journal of Agro-Environment Science 2015, 34(08):1515-21.

13. MY Z. Handbook of Rating Scales in Psychiatry. Chang sha, Hunan province, China: Hunan Science and Technology Press; 2003.

14. Buysse DJ, Reynolds CF, Monk TH, Berman SR, Kupfer DJ. The Pittsburgh Sleep Quality Index: a new instrument for psychiatric practice and research. Psychiatry Res 1989, 28(2):193-213.

15. Ali N, Hoque MA, Haque A, Salam KA, Karim MR, Rahman A, et al. Association between arsenic exposure and plasma cholinesterase activity: a population based study in Bangladesh. Environ Health 2010, 9:36.

16. WS/T211-2015: Diagnosis of endemic arsenicosis. 2015.

17. Nie X LX, Zhuo L, Cheng $Y$, Wang $H$, Zhan S. Prevalence of mild cognitive impairment in China: a metaanalysis of studies in 2001-2015. Chin J Psychiatry 2016, 49: 298-306.

18. Martinez-Finley EJ, Ali AM, Allan AM. Learning deficits in C57BL/6J mice following perinatal arsenic exposure: consequence of lower corticosterone receptor levels. Pharmacol Biochem Behav 2009, 94(2):271-7. 
19. Chan KY, Wang W, Wu JJ, Liu L, Theodoratou E, Car J, et al. Epidemiology of Alzheimer's disease and other forms of dementia in China, 1990-2010: a systematic review and analysis. Lancet 2013, 381(9882):2016-23.

20. Saedi E, Gheini MR, Faiz F, Arami MA. Diabetes mellitus and cognitive impairments. World J Diabetes 2016, 7(17):412-22.

21. Ott A, Stolk RP, van Harskamp F, Pols HA, Hofman A, Breteler MM. Diabetes mellitus and the risk of dementia: The Rotterdam Study. Neurology 1999, 53(9):1937-42.

22. Wessels AM, Lane KA, Gao S, Hall KS, Unverzagt FW, Hendrie HC. Diabetes and cognitive decline in elderly African Americans: a 15-year follow-up study. Alzheimers Dement 2011, 7(4):418-24.

23. Zhao Q, Roberts RO, Ding D, Cha R, Guo Q, Meng H, et al. Diabetes is Associated with Worse Executive Function in Both Eastern and Western Populations: Shanghai Aging Study and Mayo Clinic Study of Aging. J Alzheimers Dis 2015, 47(1):167-76.

24. Xue M, Xu W, Ou YN, Cao XP, Tan MS, Tan L, et al. Diabetes mellitus and risks of cognitive impairment and dementia: A systematic review and meta-analysis of 144 prospective studies. Ageing Res Rev, 55:100944.

25. Lennon MJ, Makkar SR, Crawford JD, Sachdev PS. Midlife Hypertension and Alzheimer's Disease: A Systematic Review and Meta-Analysis. J Alzheimers Dis 2019, 71(1):307-16.

26. Holth JK, Fritschi SK, Wang C, Pedersen NP, Cirrito JR, Mahan TE, et al. The sleep-wake cycle regulates brain interstitial fluid tau in mice and CSF tau in humans. Science 2019, 363(6429):880-4.

27. Kruger K, Straub H, Hirner AV, Hippler J, Binding N, Musshoff U. Effects of monomethylarsonic and monomethylarsonous acid on evoked synaptic potentials in hippocampal slices of adult and young rats. Toxicol Appl Pharmacol 2009, 236(1):115-23.

28. Dutta K, Prasad P, Sinha D. Chronic low level arsenic exposure evokes inflammatory responses and DNA damage. Int J Hyg Environ Health 2015, 218(6):564-74.

29. Escudero-Lourdes $C$. Toxicity mechanisms of arsenic that are shared with neurodegenerative diseases and cognitive impairment: Role of oxidative stress and inflammatory responses. Neurotoxicology 2016, 53:223-35.

30. Giasson BI, Sampathu DM, Wilson CA, Vogelsberg-Ragaglia V, Mushynski WE, Lee VM, et al. The environmental toxin arsenite induces tau hyperphosphorylation. Biochemistry 2002, 41(51):1537687.

31. Zarazua S, Burger S, Delgado JM, Jimenez-Capdeville ME, Schliebs R. Arsenic affects expression and processing of amyloid precursor protein (APP) in primary neuronal cells overexpressing the Swedish mutation of human APP. Int J Dev Neurosci 2011, 29(4):389-96.

32. Yang D, Liang C, Jin Y, Wang D. Effect of arsenic toxicity on morphology and viability of enzyme in primary culture of rat hippocampal neurons. Journal of hygiene research 2003, 32(4):309-12.

33. Sun H, Yang Y, Shao H, Sun W, Gu M, Wang H, et al. Sodium Arsenite-Induced Learning and Memory Impairment Is Associated with Endoplasmic Reticulum Stress-Mediated Apoptosis in Rat Hippocampus. Front Mol Neurosci 2017, 10:286. 


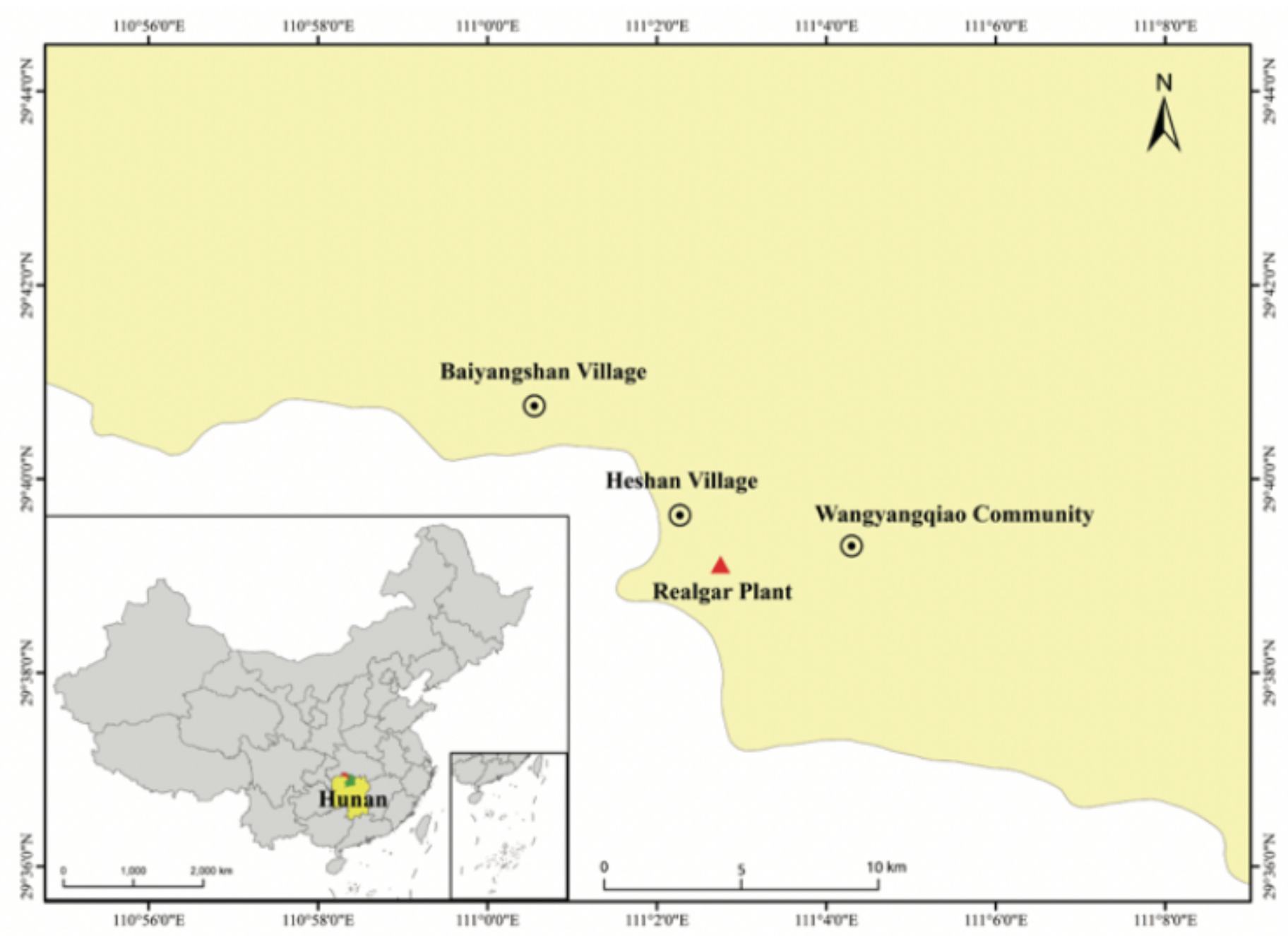

Figure 2

Geographical distribution of study locations. Note: The designations employed and the presentation of the material on this map do not imply the expression of any opinion whatsoever on the part of Research Square concerning the legal status of any country, territory, city or area or of its authorities, or concerning the delimitation of its frontiers or boundaries. This map has been provided by the authors. 

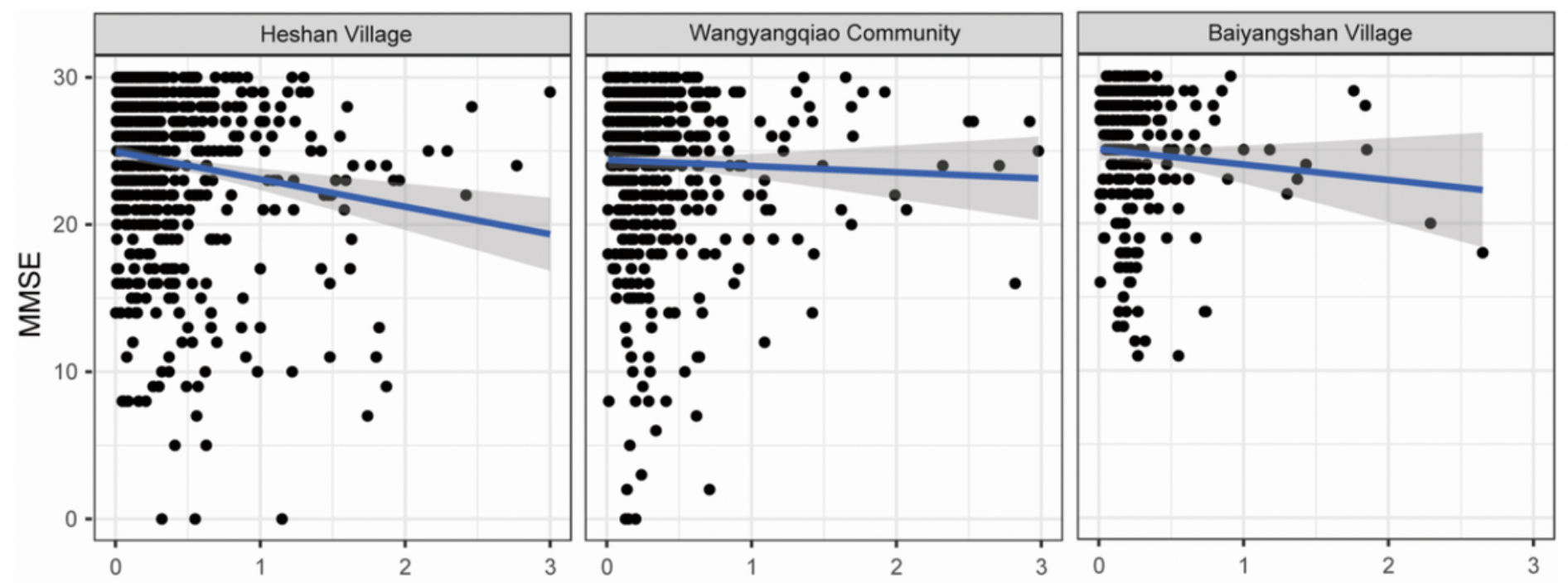

Hair arsenic concentrations (mg/kg)

Figure 3

Correlation between hair arsenic concentration and MMSE in three locations 